\title{
FATIGUE STRENGTH OF PLATE GIRDER IN BENDING CONSIDERING OUT-OF-PLANE DEFORMATION OF WEB
}

\author{
By Yukio MAEDA* and Ichiro OKURA**
}

\begin{abstract}
When a thin-walled plate girder is subjected to repeated bending, it is possible that fatigue cracks may be initiated at the toe on the web side of fillet welds connecting the compression flange to the web. They are caused by the secondary bending stress produced by out-of-plane movement of the web under repeated in-plane bending, due to its unavoidable initial deflection. In this paper, first, the relation between in-plane bending stress and secondary bending stress is formulated. Secondly, substituting the fatigue strength of fillet welds subjected to secondary bending stress into the relation, the fatigue strength for the above fatigue cracks is expressed in in-plane bending stress. It is given as a function of the web slenderness ratio, and the influence of various factors on the fatigue strength is discussed.
\end{abstract}

\section{INTRODUCTION}

As pointed out in Refs. 1) -5), when a thin-walled plate girder is subjected to repeated loading, it is possible that fatigue cracks may be initiated at the toe on the web side of fillet welds around the web panel. They are caused by the secondary bending stress at the toe on the web side of fillet welds, which is produced under an in-plane load by the out-of-plane deformation of the web with initial deflections.

In AASHTO Specification ${ }^{6}$, the limits of the web slenderness ratio of transversely stiffened plate girders are 163 in the allowable stress design and 192 in the load factor design for a steel with the yielding stress of $248.2 \mathrm{MPa}$. The use of considerably slender web plates is approved in the latter design. The limits of the web slenderness ratio in the load factor design are shown in a provision to prevent the initiation of the above-mentioned fatigue cracks. They were proposed by taking into consideration the results of fatigue tests of plate girders with transverse stiffeners ${ }^{7}$. But, they are not directly based on the fatigue strength of fillet welds subjected to secondary bending stress. On the other hand, in the case of $B S 5400^{8}$, fatigue is dealt with in its Part 10. Judging from the answer to the authors' paper ${ }^{9)}$ contributed to the conference held at University College, Cardiff in 1980, the fatigue cracks due to out-of-plane deformation of the web seems not to be considered there.

As for thin-walled plate girders in repeated bending, as pointed out in Ref.10), it is possible that the following three types of fatigue cracks may be initiated.

a) Type 1 fatigue cracks at the toe on the web side of fillet welds connecting the compression flange to the web, which are due to out-of-plane deformation of the web.

* Member of JSCE Dr. Eng., Professor, Department of Civil Engineering, Osaka University

** Member of JSCE M. Eng., Research Associate, Department of Civil Engineering, Osaka University (Yamada-Oka 2-1, Suita, Osaka 565) 
b) Type 2 fatigue cracks at the toe on the web side of fillet welds connecting the vertical stiffener to the web.

c) Type 3 fatigue cracks at fillet welds connecting the tension flange to the web.

In the case of type 2 and 3 fatigue cracks, their fatigue strengths are given ${ }^{11,122}$, and it is possible to estimate the initiation of them by comparing the flange stress calculated by the beam theory with the fatigue strength. On the other hand, in the case of type 1 fatigue cracks, the fatigue strength of fillet welds subjected to secondary bending stress is clarified ${ }^{13)}$. But, it is impossible to estimate the initiation of them based on this fatigue strength, because the relation between in-plane bending stress and secondary bending stress is not formulated. The authors have already made it clear by the finite out-of-plane deformation analysis of rectangular plates in in-plane bending by the finite element method that not only the magnitudes of the initial deflections but also their shapes will influence greatly an increase in secondary bending stress, resulting in the initiation of the type 1 fatigue cracks $^{10,14}$.

In this paper, first, the relation between load and secondary bending stress of rectangular plates in in-plane bending is formulated. Secondly, substituting the fatigue strength of fillet welds subjected to secondary bending stress into the relation, the fatigue strength of type 1 fatigue cracks is exprssed in in-plane bending stress. It is given as a function of the web slenderness ratio, and the influence of various factors on the fatigue strength is discussed.

\section{FORMULATION OF FINITE OUT-OF-PLANE DEFORMATION OF RECTANGULAR PLATES IN IN-PLANE BENDING}

(1) Relation between Load and Out-of-plane Deflection in the Case of Simple Supports at All Edges

As shown in Fig.1, a rectangular plate of $a \times b$ under in-plane bending is simply supported in the out-of-plane direction at all the edges. The initial deflection $w_{o}$ and the additional deflection $w$ are respectively assumed as follows:

$$
\begin{aligned}
& w_{o}=\sin (\pi x / a)\left\{e_{01} \sin (\pi y / b)+e_{02} \sin (2 \pi y / b)\right\}, \\
& w=\sin (\pi x / a)\left\{e_{1} \sin (\pi y / b)+e_{2} \sin (2 \pi y / b)\right\},
\end{aligned}
$$

where $e_{01}$ and $e_{02}=$ the mode components of the initial delfection, and $e_{1}$ and $e_{2}=$ the mode components of the additional deflection. Substituting Eqs.(1) and (2) into the Marguerre's plate differen-

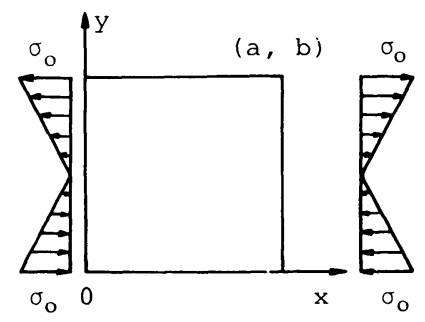

Fig. 1 Rectangular plate in in-plane bending. tial equations ${ }^{15}$, and applying the Galerkin method to them, the relation between load and out-of-plane deflection can be obtained as follows ${ }^{16)}$ :

$$
\begin{aligned}
& \frac{\sigma_{o}}{\sigma_{e}}=\frac{9 \pi^{2}}{32} \theta_{3} \frac{e_{1}}{e_{02}+e_{2}}+\frac{9 \pi^{2}}{32}\left(1-\nu^{2}\right)\left(\frac{3}{4} \theta_{5} \frac{q_{1}}{t_{w}^{2}} \frac{e_{01}+e_{1}}{e_{02}+e_{2}}+3 \theta_{7} \frac{q_{2}}{t_{w}^{2}} \frac{e_{01}+e_{1}}{e_{02}+e_{2}}+3 \theta_{8} \frac{q_{3}}{t_{w}^{2}}\right), \\
& \frac{\sigma_{o}}{\sigma_{e}}=\frac{9 \pi^{2}}{32} \theta_{4} \frac{e_{2}}{e_{01}+e_{1}}+\frac{9 \pi^{2}}{32}\left(1-\nu^{2}\right)\left(\frac{3}{4} \theta_{6} \frac{q_{2}}{t_{w}^{2}} \frac{e_{02}+e_{2}}{e_{01}+e_{1}}+3 \theta_{7} \frac{q_{1}}{t_{w}^{2}} \frac{e_{02}+e_{2}}{e_{01}+e_{1}}+3 \theta_{8} \frac{q_{3}}{t_{w}^{2}}\right),
\end{aligned}
$$

where $\sigma_{o}=$ the in-plane bending stress, $\sigma_{e}=\pi^{2} E /\left\{12\left(1-\nu^{2}\right) \beta^{2}\right\}, \beta=b / t_{w}, E=$ the Young's modulus, $\nu=$ the Poisson's ratio, $t_{w}=$ the thickness of the web plate, $\theta_{3} \sim \theta_{8}=$ the coefficients depending on the aspect ratio $a / b, q_{1}=\left(e_{01}+e_{1}\right)^{2}-e_{01}^{2}, \quad q_{2}=\left(e_{02}+e_{2}\right)^{2}-e_{02}^{2}$, and $q_{3}=\left(e_{01}+e_{1}\right)\left(e_{02}+e_{2}\right)-e_{01} e_{02}$.

(2) Relation between Load and Out-of-Plane Deflection Considering Rotational Restraint at Unloaded Edges

When a rectangular plate is subjected to in-plane bending, the out-of-plane deflection on the compressi ve side becomes larger than that on the tensile side. Accordingly, the relation between the load and the out-of plane deflection at the location of $y=b / 4$ from the compressive edge will be formulated. Here, letting $e$ denote the additional out-of-plane deflection at this location, $e$ is given by

$$
e=e_{1} / \sqrt{2}+e_{2} \text {. }
$$


Investigating each term on the right side of Eqs.(3) and (4), it is revealed that when the additional deflection is small, the respective first terms are dominative, and that as it increases, they converge into a buckling coefficient, and on the contrary, an increase in the respective second terms becomes remarkable. Considering such behavior, Eqs. ( 3 ) and ( 4 ) can be approximated by the following equation, when either of $e_{01}$ or $e_{02}$ exists for the initial deflection:

$$
\frac{\sigma_{o}}{\sigma_{e}}=k_{c r} \eta_{o}+\frac{3}{4}\left(1-\nu^{2}\right) \theta \xi_{o}
$$

where

$$
\eta_{o}=\frac{\sqrt{\left(e / t_{w}+e_{o} / t_{w}\right) e / t_{w}+r_{1}^{2}\left(e_{o} t_{w}\right)^{2}}-r_{1} e_{o} t_{w}}{e / t_{w}+e_{o} / t_{w}}, \quad \xi_{o}=\left(\frac{e}{t_{w}}+\frac{e_{o}}{t_{w}}\right)^{2}\left\{1-\left(\frac{e_{o}}{r_{2} e+e_{o}}\right)^{2}\right\},
$$

$e_{o}=e_{01} / \sqrt{2}$ or $e_{02}, k_{c r}=\left(9 \pi^{2} / 32\right) \sqrt{\theta_{3} \theta_{4}}=$ the buckling coefficient, $r_{1}=\sqrt{\theta_{3} /\left(2 \theta_{4}\right)}$ for $e_{o}=e_{01} / \sqrt{2}$ or $\sqrt{\theta_{4} /\left(8 \theta_{3}\right)}$ for $e_{0}=e_{02}$, and $\theta$ and $r_{2}=$ the coefficients.

$\eta_{0}$ can be obtained by combining Eq. ( 5 ) and the respective first terms on the right side of Eqs. ( 3 ) and (4). On the other hand, $\xi_{0}$ is assumed as above, referring to the form ${ }^{17,18)}$ of an equation of the relation between load and out-of-plane deflection of rectangular plates in compression. When $r_{2}=1, \xi_{0}$ takes the same form as the one of rectangular plates in compression. Determining the values of $\theta$ and $r_{2}$ in the same manner as mentioned in the subsequent Section 2. (5) for the relation between load and out-of-plane deflection given by Eqs.( 3 ) and (4), substituting these values into Eq.(6), and comparing Eq.(6) with Eqs.( 3 ) and (4), Eq.(6) approximates Eqs.(3) and (4) very well.

If the values of $k_{c r}, \theta, r_{1}$ and $r_{2}$, which are determined by taking into account the rotational restraint at the unloaded edges, are used, Eq. (6) will give the relation between load and out-of-plane deflection considering the rotational restraint at the unloaded edges. A method to determine the values of these coefficients is stated in the subsequent sections.

(3) Determination of $k_{c r}$

The differential equation for the buckling analysis of plates in in-plane bending is as follows:

$$
\frac{D_{w}}{t_{w}} \nabla^{4} w+\sigma_{o}\left(1-2 \frac{y}{b}\right) \frac{\partial^{2} w}{\partial x^{2}}=0,
$$

where $D_{w}=E t_{w}^{3} /\left\{12\left(1-\nu^{2}\right)\right\}$ and $\nabla^{4}=\partial^{4} / \partial x^{4}+2 \partial^{4} / \partial x^{2} \partial y^{2}+\partial^{4} / \partial y^{4}$. The following equation is assumed for the additional deflection:

$w=Y(y) \sin (\pi x / a)$

where $Y(y)=$ the function of $y$. Substituting Eq.( 8$)$ into Eq.( 7$)$, and eliminating $\sin (\pi x / a)$, an ordinary differential equation about the function $Y(y)$ is obtained. Transforming this equation into finite-difference equations, and solving numerically the eigenvalue problem defined by the equations, $k_{c r}$ is determined.

(4) Determination of $r_{1}$

The first term on the right side of Eq.( 6 ) can also be obtained by substituting Eqs.( 1 ) and (2) into the following equation ${ }^{19)}$ and by applying the Galerkin method to it:

$$
\frac{D_{w}}{t_{w}} \nabla^{4} w=-\sigma_{o}\left(1-2 \frac{y}{b}\right) \frac{\partial^{2}\left(w_{o}+w\right)}{\partial x^{2}} \text {. }
$$

Substituting Eqs.( 1 ) and ( 8 ) into Eq.(9), and eliminating $\sin (\pi x / a)$, an ordinary differential equation about the function $Y(y)$ is obtained. Transforming this equation into finite-difference equations, and solving the simultaneous linear equations made by the equations, the relation between load and out-of-plane deflection is obtained.

On the other hand, solving $\sigma_{o} / \sigma_{e}=k_{c r} \eta_{o}$ about $r_{1}$, the following equation is obtained :

$$
r_{1}=\frac{\frac{e}{e_{o}}-\left(\frac{1}{k_{c r}} \frac{\sigma_{o}}{\sigma_{e}}\right)^{2}\left(\frac{e}{e_{o}}+1\right)}{\frac{2}{k_{c r}} \frac{\sigma_{o}}{\sigma_{e}}} \text {. }
$$


Substituting the above-mentioned relation between load and out-of-plane deflection into Eq.(10), $r_{1}$ is almost constant, and the value at $e / e_{o}=1$ may be used for $r_{1}$.

(5) Determination of $\theta$ and $r_{2}$

To determine the values of $\theta$ and $r_{2}$, the results of the finite element method analysis ${ }^{10,14)}$ will be used. A mesh division of a rectangular plate is shown in Fig.2. Considering symmetry of the plate, its half is divided into 144 elements. The unloaded edges are free to move in the in-plane direction, and for boundary conditions in the out-of-plane direction at these edges, the following cases are treated :

( I ) both edges simply supported, and

(II) both edges fixed.

The loaded edges are simply supported in the out-of-plane direction, and the in-plane bending is applied so that the membrane stress varies linearly at these edges. Substituting the values of $\sigma_{o} / \sigma_{e}$ and $e / t_{w}$ obtained by the finite element method analysis into the terms of $\sigma_{o} / \sigma_{e}-k_{c r} \eta_{o}$ and $\xi_{o}$, the relation between the two terms is obtained. It becomes almost linear at a certain value of $r_{2}$ in $\xi_{o}$, regardless of the magnitudes of the initial deflections, and the values of $\theta$ are determined by applying the least squares method to it.

The values of $k_{c r}, r_{1}, r_{2}$ and $\theta$ are shown in Table 1. The curves of load versus out-of-plane deflection given by Eq. ( 6 ) and the results of the finite element method analysis are shown in Fig.3. It is seen that Eq. (6) is very close to the values of the finite element method analysis.

(6) Relation between Secondary Bendidng Stress and Out-of-Plane Deflection

The relation between secondary bending stress $\sigma_{b} / \sigma_{e}$ at the point $(a / 2,0)$ in Fig. 1 and out-of-plane deflection is assumed as follows, and the acceptability of this equation will be explained :

$$
\sigma_{b} / \sigma_{e}=S \eta_{b}+\sqrt{A^{2} \xi_{b}^{2}+B^{2}}-B,
$$

where

$$
\eta_{b}=\frac{\left(1+2 r_{1}\right) \eta_{o}+r_{3}\left(1-\eta_{o}\right)}{\eta_{o}+2 r_{1}} \frac{e}{t_{w}}, \quad \xi_{b}=\left(\frac{e}{t_{w}}+\frac{e_{0}}{t_{w}}\right)^{2}\left\{1-\left(\frac{e_{o}}{r_{4} e+e_{o}}\right)^{2}\right\},
$$

and $S, r_{3}, r_{4}, A$ and $B=$ the coefficients.

The first term on the right side of Eq.(11) is obtained as follows. The relation between secondary bending stress and out-of-plane deflection is given by

$$
\sigma_{b} / \sigma_{e}=C_{1} e_{1} / t_{w}+C_{2} e_{2} / t_{w}
$$

where $C_{1}$ and $C_{2}=$ the coefficients. Eliminating $e_{1}$ and $e_{2}$ in Eq.(12) by Eq.( 5$)$ and the respective first terms on the right side of Eqs.( 3$)$ and (4), the following equation is obtained when $e_{01}$ only exists :

$$
\frac{\sigma_{b}}{\sigma_{e}}=\frac{\sqrt{2} C_{1} \eta_{o}+2 C_{2} r_{1}}{\eta_{o}+2 r_{1}} \frac{e}{t_{w}} \text {. }
$$

Here, let $S$ denote the coefficient in front of $e / t_{w}$ in Eq.(13) in the case of $e_{01} / t_{w}=0$, and let $r_{3}=2 r_{1} C_{2} / S$. Eliminating $C_{1}$ and $C_{2}$ in Eq.(13) with these coefficients, the first term on the right side of

\begin{tabular}{|c|c|c|c|c|c|}
\hline & \multicolumn{3}{|c|}{ (I) } & \multicolumn{2}{|c|}{ (II) } \\
\hline $\bar{a} / \mathrm{b}$ & $1 / 3$ & 0.5 & 1.0 & $1 / 3$ & 0.5 \\
\hline$e_{0}$ & $\mathbf{e}_{01} / \sqrt{2} \quad \mathbf{e}_{02}$ & $\mathbf{e}_{01} / \sqrt{2} \quad e_{02}$ & $\mathbf{e}_{01} / \sqrt{2} \quad \mathbf{e}_{02}$ & $\mathbf{e}_{01} / \sqrt{2} \mathbf{e}_{02}$ & $\mathbf{e}_{01} / \sqrt{2} \quad \mathbf{e}_{0}$ \\
\hline$\overline{\mathrm{k}_{\mathrm{cr}}}$ & 33.78 & 25.63 & 27.13 & 43.72 & 39.46 \\
\hline $\mathbf{r}_{1}$ & $0.44 \quad 0.50$ & $0.41 \quad 0.61$ & $0.28 \quad 0.90$ & 0.52 & $0.46 \quad 0.64$ \\
\hline $\mathbf{r}_{2}$ & $0.35 \quad 0.60$ & $0.65 \quad 1.0$ & 0.35 & $0.35 \quad 0.50$ & $0.65 \quad 0.64$ \\
\hline$\theta$ & 5.07 & 5.25 & 6.52 & 6.51 & 8.01 \\
\hline $\mathrm{S}$ & & & & 86.69 & 62.31 \\
\hline$r_{3}$ & & & & $1.03 \quad 1.28$ & $1.08 \quad 1.36$ \\
\hline$r_{4}$ & & & & 0.6 & 1.3 \\
\hline A & & & & 11.70 & 6.14 \\
\hline B & & & & 5.29 & 1.65 \\
\hline
\end{tabular}

Table 1 Values of coefficients.

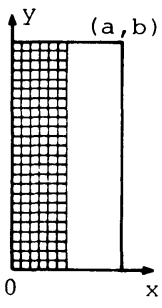

Fig. 2 Mesh division.
(I) Both edges simply supported

(II) Both edges fixed 

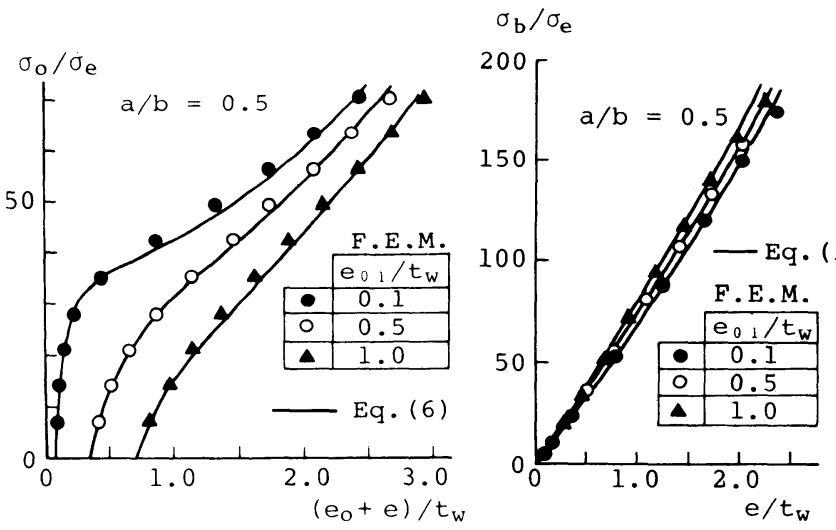

Fig. $3 \sigma_{o} / \sigma_{e}$ versus $\left(e_{o}+e\right) / t_{w}$ [Case (II)]. Fig. $4 \sigma_{b} / \sigma_{e}$ versus $e / t_{w}[\mathrm{Case}(\mathrm{II})]$.

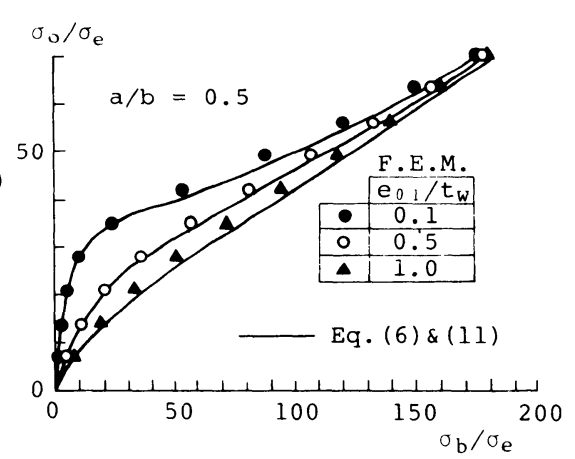

Fig. $5 \sigma_{0} / \sigma_{e}$ versus $\sigma_{b} / \sigma_{e}[$ Case (II)].

Eq.(11) is obtained. On the other hand, when $e_{02}$ only exists, such a term is obtained in the same manner. The coefficient $S$ is, from the definition, an increase in secondary bending stress per a unit out-of-plane deflection at $y=b / 4$ of the buckled form, and its values are determined by the buckling analysis in Section 2. (3).

The coefficient $r_{3}$ is determined by solving $\sigma_{b} / \sigma_{e}=S \eta_{b}$ about $r_{3}$ as follows:

$$
r_{3}=\frac{(1 / S)\left(\sigma_{b} / \sigma_{e}\right)}{\left(e / e_{o}\right)\left(e_{o} / t_{w}\right)} \frac{\eta_{o}+2 r_{1}}{1-\eta_{o}}-\left(1+2 r_{1}\right) \frac{\eta_{o}}{1-\eta_{o}} \text {. }
$$

Substituting the relation between secondary bending stress and out-of-plane deflection obtained in Section

2. (4) into Eq.(14), $r_{3}$ is almost constant, and the value at $e / e_{o}=1$ may be used for $r_{3}$.

The coefficients $r_{4}, A$ and $B$ are determined as follows. Substituting the values of $\sigma_{b} / \sigma_{e}$ and $e / t_{w}$ obtained by the finite element method analysis into the terms of $\sigma_{b} / \sigma_{e}-S \eta_{b}$ and $\xi_{b}$, the relation between the two terms is obtained. It can be expressed in a curved line at a certain value of $r_{4}$ in $\xi_{b}$, regardless of the magnitudes of the initial deflections, and the values of $A$ and $B$ are determined by applying the least squares method to it.

The values of the coefficients $S, r_{3}, r_{4}, A$ and $B$ are also shown in Table 1. The comparison of $\sigma_{b} / \sigma_{e}$ versus $e / t_{w}$ curves given by Eq.(11) with the results of the finite element method analysis is shown in Fig.4. The relation between $\sigma_{o} / \sigma_{e}$ and $\sigma_{b} / \sigma_{e}$ is obtained by Eqs.( 6$)$ and (11). Its comparison with the results of the finite element method analysis is shown in Fig.5.

\section{RELATION BETWEEN LOAD AND SECONDARY BENDING STRESS CONSIDER- ING BOTH INITIAL DEFLECTION AND RESIDUAL STRESS}

\section{(1) Relation between Load and Out-of-Plane Deflection Considering Both Initial Deflection and Residual Stress}

The fundamental plate differential equations for the finite out-of-plane deformation of thin plataes which have the initial deflection and the residual stress together, are given as follows ${ }^{20)}$ :

$$
\begin{aligned}
& \frac{1}{E} \nabla^{4} F=\left\{\frac{\partial^{2}\left(w_{o}+w\right)}{\partial x \partial y}\right\}^{2}-\frac{\partial^{2}\left(w_{o}+w\right)}{\partial x^{2}} \frac{\partial^{2}\left(w_{o}+w\right)}{\partial y^{2}}-\left\{\left(\frac{\partial^{2} w_{o}}{\partial x \partial y}\right)^{2}-\frac{\partial^{2} w_{o}}{\partial x^{2}} \frac{\partial^{2} w_{o}}{\partial y^{2}}\right\}, \ldots \ldots \ldots . . . \\
& \frac{D_{w}}{t_{w}} \nabla^{4} w=\frac{\partial^{2}\left(F_{o}+F\right)}{\partial y^{2}} \frac{\partial^{2}\left(w_{o}+w\right)}{\partial x^{2}}-2 \frac{\partial^{2}\left(F_{o}+F\right)}{\partial x \partial y} \cdot \frac{\partial^{2}\left(w_{o}+w\right)}{\partial x \partial y}+\frac{\partial^{2}\left(F_{o}+F\right)}{\partial x^{2}} \frac{\partial^{2}\left(w_{o}+w\right)}{\partial y^{2}},
\end{aligned}
$$

where $F=$ the stress function and $F_{o}=$ the stress function for residual stress. Eqs.(15) and (16) show that the initial deflection and residual stress interact each other. Generally, the initial deflections specified in a specification for bridges or measured in fatigue tests are considered to be the ones at the time when the 
equilibrium condition is reached after the initial deflection and residual stress have interacted each other. The fundamental plate differential equations defined by such initial deflections for the finite out-of-plane deformation of thin plates will be derived in the next place.

Let $w_{r}$ and $F_{r}$ respectively denote the out-of-plane deflection and stress function at the time when the equilibrium condition is reached after the initial deflectioin has been varied by the residual stress. Furthermore, let $w_{a}$ and $F_{a}$ respectively denote the additional out-of-plane deflectioin and stress function for a stress produced by loading. Using these parameters, $w$ and $F$ are expressed as follows:

$$
\begin{aligned}
& w=w_{a}+w_{r}-w_{o}, \\
& F=F_{a}+F_{r}-F_{o} .
\end{aligned}
$$

Substituting Eqs.(17) and (18) into Eqs.(15) and (16), and eliminating $w_{o}$ and $F_{o}$ by using the relation that Eqs.(15) and (16) also hold on the unloaded condidtions of $w_{a}=0$ and $F_{a}=0$, Eqs.(15) and (16) are respectively transformed as follows :

$$
\begin{aligned}
& \frac{1}{E} \nabla^{4} F_{a}=\left\{\frac{\partial^{2}\left(w_{r}+w_{a}\right)}{\partial x \partial y}\right\}^{2}-\frac{\partial^{2}\left(w_{r}+w_{a}\right)}{\partial x^{2}} \frac{\partial^{2}\left(w_{r}+w_{a}\right)}{\partial y^{2}}-\left\{\left(\frac{\partial^{2} w_{r}}{\partial x \partial y}\right)^{2}-\frac{\partial^{2} w_{r}}{\partial x^{2}} \frac{\partial^{2} w_{r}}{\partial y^{2}}\right\}, \\
& \frac{D_{w}}{t_{w}} \nabla^{4} w_{a}-\left(\frac{\partial^{2} F_{r}}{\partial y^{2}} \frac{\partial^{2} w_{a}}{\partial x^{2}}-2 \frac{\partial^{2} F_{r}}{\partial x \partial y} \frac{\partial^{2} w_{a}}{\partial x \partial y}+\frac{\partial^{2} F_{r}}{\partial x^{2}} \frac{\partial^{2} w_{a}}{\partial y^{2}}\right) \\
& =\frac{\partial^{2} F_{a}}{\partial y^{2}} \frac{\partial^{2}\left(w_{r}+w_{a}\right)}{\partial x^{2}}-2 \frac{\partial^{2} F_{a}}{\partial x \partial y} \frac{\partial^{2}\left(w_{r}+w_{a}\right)}{\partial x \partial y}+\frac{\partial^{2} F_{a}}{\partial x^{2}} \frac{\partial^{2}\left(w_{r}+w_{a}\right)}{\partial y^{2}}
\end{aligned}
$$

Seemingly, Eqs.(19) and (20) show that the terms on the residudal stress marked with a wave line are added to the Marguerre's fundamental plate differential equations defined by the initial deflection $w_{r}$ and the additional out-of-plane deflection $w_{a}$. If the initial deflection of Eq.( 1 ), the additional deflection of Eq. ( 2 ) and the residual stress of rectangular distribution such as shown in Fig.6 are assumed for $w_{r}, w_{a}$ and $F_{r}$, respectively, $\theta_{3}$ in Eq. ( 3 ) and $\theta_{4}$ in Eq. (4) have only to be replaced by $\theta_{3}-\{\sin (2 \pi c / b) /(2 \pi c / b)\} \sigma_{r c}$ $/ \sigma_{e}$ and $\theta_{4}-\{\sin (4 \pi c / b) /(4 \pi c / b)\} \sigma_{r c} / \sigma_{e}$, respectively. These coefficients are related only to $k_{c r}$ and $r_{1}$ in Eq. $(6)$.

When $F_{r}$ is generally given by a function of only $y, k_{c r}$ and $r_{1}$ are respectively determined as follows. $k_{c r}$ is determined by the following equation instead of Eq. $(7)$ :

$$
\frac{D_{w}}{t_{w}} \nabla^{4} w_{a}-\left\{\frac{\partial^{2} F_{r}}{\partial y^{2}}-\sigma_{o}\left(1-2 \frac{y}{b}\right)\right\} \frac{\partial^{2} w_{a}}{\partial x^{2}}=0
$$

$r_{1}$ is determined by substituting the relation between load and out-of-plane deflection obtained by the following equation instead of Eq.(9) into Eq.(10) :

$$
\frac{D_{w}}{t_{w}} \nabla^{4} w_{a}-\frac{\partial^{2} F_{r}}{\partial y^{2}} \frac{\partial^{2} w_{a}}{\partial x^{2}}=-\sigma_{o}\left(1-2 \frac{y}{b}\right) \frac{\partial^{2}\left(w_{r}+w_{a}\right)}{\partial x^{2}}
$$

The values of $k_{c r}$ and $r_{1}$ obtained by Eqs. (21) and (22) for the residual stress shown in Fig.6 are listed in Table 2. It is seen that as the residual stress increases, $k_{c r}$ decreases, and that $r_{1}$ varies depending on the magnitude of the residual stress.

(2) Relation between Secondary Bending Stress and Out-of-Plane Deflection Considering Both Initial Deflection and Residual Stress

The values of $S$ considering the residual stress are determined by Eq.(21), and the values of $r_{3}$ are determined by substituting the relation between secondary bending stress and out-of-plane deflection obtained by Eq.(22) into Eq.(14). The values of $S$ and $r_{3}$ for the residual stress shown in Fig.6 are listed in Table 2. It is seen that as the residual stress increases, $S$ decreases, and that $r_{3}$ varies depending on the magnitude of the residual stress.

The influence of the residual stress on the coefficients $A, B$ and $r_{4}$ will be examined by the results of the finite element method analysis. In the finite element method analysis program ${ }^{17)}$ considering the residual stress, after the equilibrium condition has been calculated for a given initial deflection and a given residual 


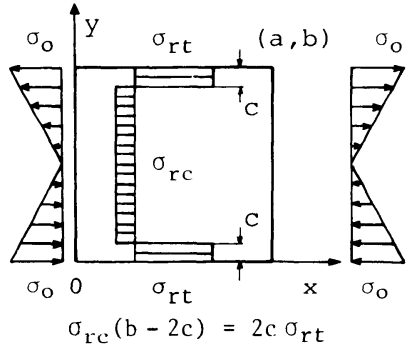

Fig. 6 Rectangular plate in in-plane bending with residual stress.

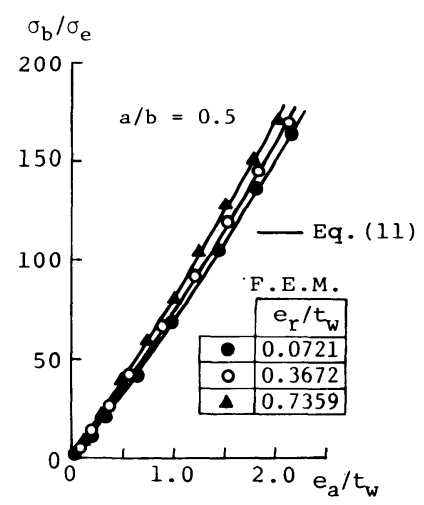

Fig. $7 \sigma_{b} / \sigma_{e}$ versus $e_{a} / t_{w}[$ Case(II)].

Table 2 Influence of residual stress on $k_{c r}, S, r_{1}$ and $r_{3}[$ Case(II)].

for $a / b=1 / 3$

\begin{tabular}{c|c|c|cc|cc}
\hline \multirow{2}{*}{$\sigma_{r_{c} / \sigma_{e}}$} & \multirow{3}{*}{$k_{c \cdot r}$} & & \multicolumn{4}{|c}{$\mathrm{e}_{\mathrm{o}}$} \\
\cline { 4 - 7 }$(\mathrm{c} / \mathrm{h}=1 / 12)$ & & \multicolumn{3}{|c|}{$\mathrm{e}_{01} / \sqrt{2}$} & \multicolumn{2}{|c}{$\mathrm{e}_{02}$} \\
\cline { 4 - 7 } & & $r_{1}$ & $r_{3}$ & $r_{1}$ & $r_{3}$ \\
\hline 0 & 43.72 & 86.69 & 0.52 & 1.03 & 0.57 & 1.28 \\
2 & 39.06 & 81.02 & 0.51 & 1.05 & 0.58 & $1.30)$ \\
4 & 34.11 & 75.36 & 0.49 & 1.06 & 0.60 & 1.33 \\
6 & 28.76 & 69.59 & 0.46 & 1.05 & 0.63 & 1.36 \\
\hline
\end{tabular}

For $a / b=0.5$

\begin{tabular}{c|c|c|cc|cc}
\hline \multirow{2}{*}{$\begin{array}{c}\sigma_{\mathrm{rc}} / \sigma_{\mathrm{e}} \\
(\mathrm{c} / \mathrm{b}=1 / 12)\end{array}$} & \multirow{3}{*}{$\mathrm{k}_{\mathrm{cr}}$} & $\mathrm{s}$ & \multicolumn{4}{|c}{$\mathrm{e}_{\mathrm{o}}$} \\
\cline { 4 - 7 } & & & \multicolumn{2}{|c}{$\mathrm{e}_{01} / \sqrt{2}$} & \multicolumn{2}{|c}{$\mathrm{e}_{02}$} \\
\cline { 4 - 7 } & & $\mathrm{r}_{1}$ & $\mathrm{r}_{3}$ & $\mathrm{r}_{1}$ & $\mathrm{r}_{3}$ \\
\hline 0 & 39.46 & 62.31 & 0.46 & 1.08 & 0.64 & 1.36 \\
2 & 33.17 & 58.70 & 0.42 & 1.02 & 0.68 & 1.40 \\
4 & 26.07 & 54.66 & 0.36 & 0.92 & 0.78 & 1.53 \\
6 & 17.26 & 49.60 & 0.26 & 0.72 & 1.04 & 1.94 \\
\hline
\end{tabular}

stress, the in-plane bending is applied. In the case of $c / b=1 / 12$ and $\sigma_{r c} / \sigma_{e}=4$ for the residual stress of rectangular distribution shown in Fig.6, calculations are carried out for the initial deflections of $e_{01} / t_{w}=0.1,0.5$ and 1.0. The aspect ratio $a / b$ is 0.5 in each case. Letting $e_{r}$ denote the out-of-plane deflection at $y=b / 4$ at the time when the equilibrium condition is calculated for these residual stress and initial deflections, $e_{r} / t_{w}$ becomes $0.0721,0.3672$ and 0.7359 for $e_{01} / t_{w}=0.1,0.5$ and 1.0 , respectively. Letting $e_{a}$ denote the additional out-of-plane deflection at $y=b / 4$ produced by loading of in-plane bending, the relation between $\sigma_{b} / \sigma_{e}$ and $e_{a} / t_{w}$ is obtained as shown in Fig.7. Replacing $e_{o}$ and $e$ in Eq.(11) with $e_{r}$ and $e_{a}$, respectively, the relation this equation gives is also shown in the figure. The values on the column of $a / b=0.5$ and $e_{o}=e_{01} / \sqrt{2}$ in Case (II) in Table 1 are used for the coefficients $S, A, B, r_{1}, r_{3}$ and $r_{4}$. It is seen from Fig.7 that Eq.(11) is very close to the values of the finite element method analysis. Accordingly, it is concluded that the residual stress does not influence the coefficients $A, B$ and $r_{4}$. Also, the term of $S \eta_{b}$ in Eq.(11) seems not to be much influenced by the residual stress, though the coefficients $S$ and $r_{3}$ are influenced by the residual stress.

From the above discussion, to apply Eqs.(6) and (11) to the case the residual stress also exists, the out-of-plane deflection at the time when the equilibrium condition is reached after the initial deflection has been varied by the residual stress has only to be used for $e_{o}$, and the coefficients $k_{c r}$ and $r_{1}$ considering the residual stress have only to be used.

\section{FATIGUE STRENGTH CONSIDERING OUT-OF-PLANE DEFORMATION OF WEB}

\section{(1) Influence of Initial Deflection Shape}

The authors revealed in Refs.10), 14) that not only the magnitudes of the initial deflections but also their shapes would influence the initiation of type 1 fatigue cracks. In the case of the web panels of aspect ratio $a / b=1$, the initial deflection of mode 1 in the $x$-directioin does not much increase the secondary bending stress, but the ones of mode 2 and 3 do it greatly. Here, the mode 1,2 and 3 mean the shapes of one, two and three half-sine-waves, respectively. On the other hand, as for the initial deflection shapes in the $y$-directiion, the mode 2 makes the largest secondary bending stress. For web panels which have the initial deflection of mode 2 or 3 in the $x$-direction, the relation between in-plane bending stress $\sigma_{o}$ and secondary bending stress $\sigma_{b}$ can be calculated by Eqs. ( 6 ) and (11). The values on columns of $a / b=0.5$ and $1 / 3$ in Case (II) in Table 1 are used in these equations corresponding to the initial deflections of mode 2 and 3 , 


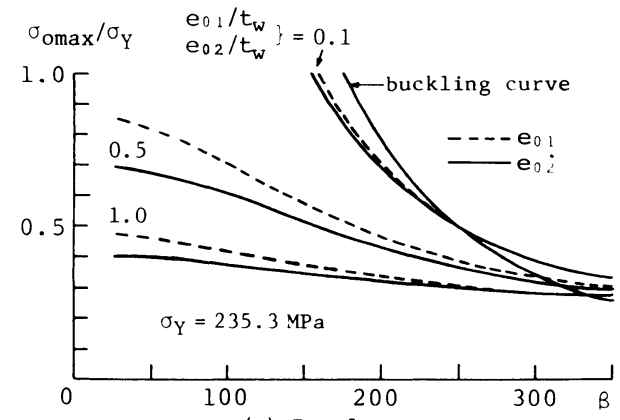

(a) $\mathrm{R}=0$

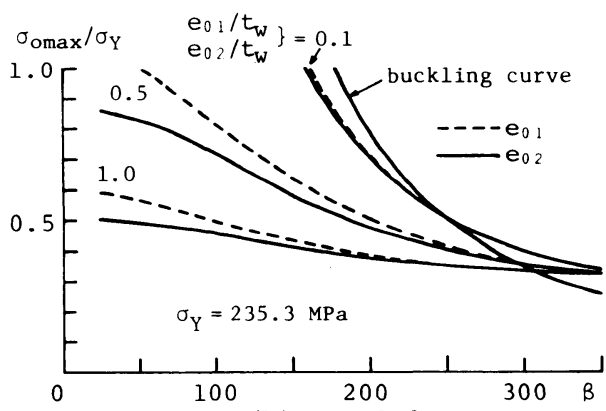

(b) $\mathrm{R}=0.2$

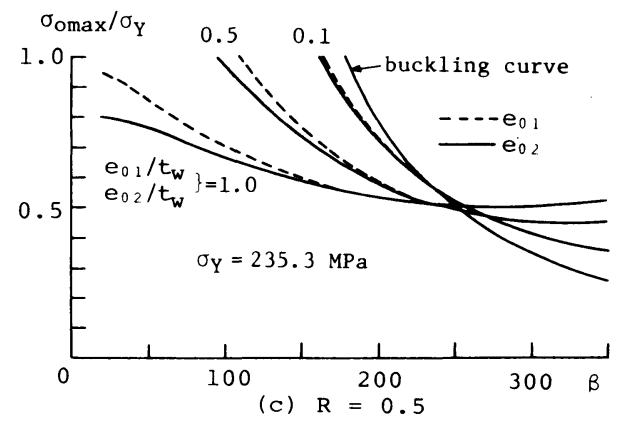

Fig. $8 \sigma_{\text {omax }} / \sigma_{Y}$ versus $\beta$ for $a / b=0.5$.



Fig. $9 \sigma_{\text {omax }} / \sigma_{Y}$ versus $\beta$ for $a / b=0.5$ and $1 / 3$.

respectively.

The fatigue strength ${ }^{13)}$ for the secondary bending stress range at $2 \times 10^{6}$ cycles is about $166.7 \mathrm{MPa}$ for steels of JIS-SS41, which have the minimum specified tensile strength of $402 \mathrm{MPa}$. Letting $\sigma_{\mathrm{bmin}}$ and $\sigma_{\mathrm{bmax}}$ denote the secondary bending stresses corresponding to the minimum and maximum in-plane bending stresses $\sigma_{\text {omin }}$ and $\sigma_{\text {omax }}$, respectively, and solving Eqs.(6) and (11) under the condition of

$$
\sigma_{\mathrm{bmax}}-\sigma_{\mathrm{bmin}}=166.7 \text {, }
$$

the relatioin between the maximum in-plane bending stress $\sigma_{\text {omax }}$ for the $2 \times 10^{6}$ cycles fatigue strength and the web slenderness ratio $\beta$ can be obtained.

The relation between $\sigma_{\mathrm{omax}} / \sigma_{Y}$ and $\beta$ in the case of $a / b=0.5$ is shown in Fig.8. Here, $\sigma_{Y}$ is the specified yielding stress, which is $235.3 \mathrm{MPa}$ for SS41 steels. $R$ is the in-plane bending stress ratio defined by the following equation :

$$
R=\sigma_{\mathrm{omin}} / \sigma_{\mathrm{omax}}
$$

From the figure, the followings are pointed out:

1) The difference of $\sigma_{\mathrm{omax}} / \sigma_{Y}$ due to the components $e_{01}$ and $e_{02}$ of the initial deflection mode in the $y$-direction becomes smaller with an increase of the web slenderness ratio $\beta$.

2) $\sigma_{\text {omax }} / \sigma_{Y}$ becomes larger with an increase of $R$. In the case of $R=0.5$, after $\beta$ has exceeded by about 250, $\sigma_{\text {omax }} / \sigma_{Y}$ for a large initial deflection becomes larger than that for a small initial deflection. Furthermore, $\sigma_{\text {omax }} / \sigma_{Y}$ for $e_{01} / t_{w}=1.0$ in the case of $R=0.5$ starts to rise after $\beta$ has exceeded by about 300. As $\beta$ increases, the web becomes more slender and the out-of-plane deflection increases. With an increase in the out-of-plane deflection, the relation between $\sigma_{o}$ and $\sigma_{b}$ obtained by Eqs. (6) and (11) converges to the one given by

$$
\sigma_{o}=(3 / 4)\left(1-\nu^{2}\right)(\theta / A) \sigma_{b} \text {. }
$$

This equation gives $\sigma_{\mathrm{omax}} / \sigma_{Y}=0.63,0.78$ and 1.26 for $R=0,0.2$ and 0.5 under the condition of Eq.(23), respectively. Accordingly, after $\sigma_{\mathrm{omax}} / \sigma_{Y}$ has decreased with an increase of $\beta$ and has reached a minimum value, it increases and converges to a limitting value given by Eq.(25).

3) The buckling curve given by the following equation is also shown in Fig. 8 :

$$
\sigma_{\text {omax }}=39.46 \sigma_{e} \text {. }
$$

There exists the region of $\beta$ where $\sigma_{\mathrm{omax}} / \sigma_{Y}$ for the $2 \times 10^{6}$ cycles fatigue strength is much smaller than the 
buckling strength, depending on the magnitude of the initial deflection and stress ratio. For example, for $\beta$ $=200$, the buckling strength is $\sigma_{\text {omax }} / \sigma_{Y}=0.78$, but the $2 \times 10^{6}$ cycles fatigue strength at $R=0$ is $\sigma_{\text {omax }} / \sigma_{Y}$ $=0.69,0.43$ and 0.32 for $e_{02} / t_{w}=0.1,0.5$ and 1.0 , respectively.

The relation between $\sigma_{\mathrm{omax}} / \sigma_{Y}$ and $\beta$ in the case of $a / b=1 / 3$ shows the same tendency as that in the case of $a / b=0.5$. However, as shown in Fig.9, $\sigma_{\mathrm{omax}} / \sigma_{\mathrm{Y}}$ for $a / b=1 / 3$ is always smaller than that for $a / b=0.5$ except for the case of $e_{02} / t_{w}=0.1$. In the case of $e_{02} / t_{w}=0.1, \sigma_{\text {omax }} / \sigma_{Y}$ for $a / b=1 / 3$ is slightly larger than that for $a / b=0.5$ in the region of $\beta$ less than about 250 .

\section{(2) Influence of Residual Stress}

As the web becomes thinner, the rotational restraint of the flanges to the web becomes larger, and the web can be regarded as fix-supported by the flanges ${ }^{10}$. On the other hand, in actual plate girders, there exists the residual stress in their webs due to welding. As mentioned in Chapter 3 , the residual stress makes the buckling coefficient $k_{c r}$ in Eq. (6) decrease. Accordingly, the influence of the residual stress on the fatigue strength will be examined by decreasing the value of $k_{c r}$ from the value for the fixed support at the unloaded edges. For the coefficients in Eqs. (6) and (11) except $k_{c r}$, the values of Case (II) in Table 1 are used.
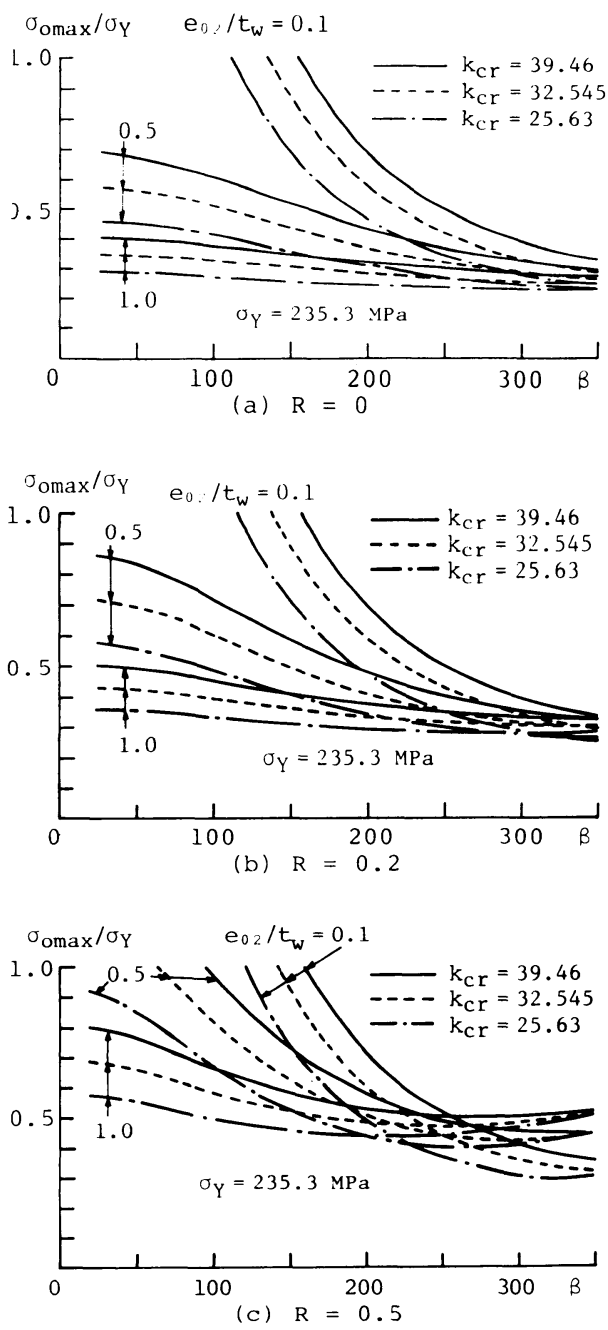

Fig. 10 Influence of residual stress $[a / b=0.5]$.
The relation between $\sigma_{\mathrm{omax}} / \sigma_{Y}$ and $\beta$ in the case of $a / b=0.5$ is shown in Fig.10. $k_{c r}=39.46$ and 25.63 in the figure correspond to the values of the buckling coefficient for the fixed and simple supports at the unloaded edges, respectively, and $k_{c r}=32.545$ is the mean value of the two. It is seen that as $k_{c r}$ decreases, $\sigma_{o \max } / \sigma_{Y}$ also does. The degree of the decrease of $\sigma_{\text {omax }} / \sigma_{Y}$ due to the residudal stress becomes smaller with the increase of $R$ and $\beta$. Especially, the curves for $e_{02} / t_{w}=0.5$ and 1.0 at $R=0.5$ approach each other and rise with the increase of $\beta$. This is because they fast converge to the values given by Eq.(25) which is not related to $k_{c r}$.

\section{(3) Influence of Yielding Stress}

It is revealed by the results of fatigue tests of T-type fillet welds subjected to repeated out-ofplane bending that the $2 \times 10^{6}$ cycles fatigue strength of SM58 steels, which have the minimum specified tensile strength of $569 \mathrm{MPa}$, is almost the same as the one of SS41 steels ${ }^{13}$. Accordingly, for steels of the yielding stress not exceeding $451.1 \mathrm{MPa}$ which is the

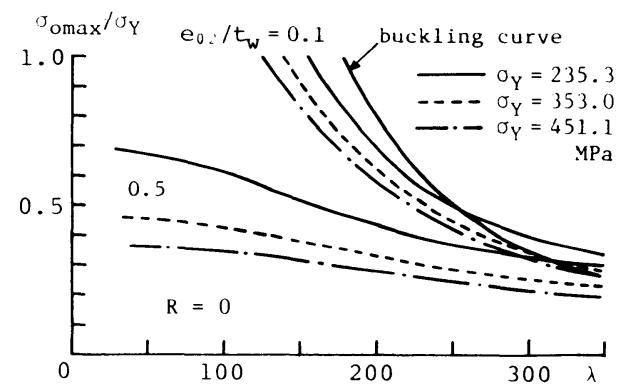

Fig. 11 Influence of yielding stress $[a / b=0.5]$. 
specified yielding stress of SM58, the condition of Eq.(23) will be used.

The relation between $\sigma_{\mathrm{omax}} / \sigma_{Y}$ and $\lambda$ in the case of $a / b=0.5$ and $R=0$ is shown in Fig.11. Here, $\lambda$ is defined as follows :

$$
\lambda=\beta \sqrt{\sigma_{Y} / 235.3}
$$

It is seen from the figure that $\sigma_{\mathrm{omax}} / \sigma_{Y}$ decreases with the increase of the yielding stress. This can be explained as follows.

Using Eq.(27), the terms $\sigma_{o} / \sigma_{e}$ and $\sigma_{b} / \sigma_{e}$ on the left side of Eqs.( 6$)$ and (11) are transformed into 1.264 $\times 10^{-3} \lambda^{2}\left(\sigma_{o} / \sigma_{Y}\right)$ and $1.264 \times 10^{-3} \lambda^{2}\left(\sigma_{b} / \sigma_{Y}\right)$, respectively. The range of $\sigma_{b} / \sigma_{Y}$ for the $2 \times 10^{6}$ cycles fatigue strength is $166.7 / \sigma_{Y}$ from Eq.(23). Since $166.7 / \sigma_{Y}$ decreases with the increase of $\sigma_{Y}, \sigma_{\text {omax }} / \sigma_{Y}$ also decreases.

Generally, using the web slenderness ratio multiplied by the square root of the yielding stress such as Eq.(27), the buckling strength can be expressed independently of the yielding stress. Even if such a parameter is used, however, the relation between fatigue strength and web slenderness ratio cannot be expressed independently of the yielding stress.

\section{CONCLUSIONS}

The relation between load and secondary bending stress of rectangular plates in in-plane bending was given by Eqs.( 6 ) and (11). Using these equations, the fatigue strength of type 1 fatigue cracks due to out-of-plane deformation of the webs of thin-walled plate girders subjected to repeated bending was expressed in in-plane bending stress. This fatigue strength was given as a function of the web slenderness ratio, and the following features were pointed out.

(1) The influence of the initial deflection mode in the $y$-direction on the fatigue strength becomes smaller with the increase of the web slenderness ratio.

(2) As the stress ratio increases, the fatigue strength also increases. When the stress ratio is small, the fatigue strength for a large initial deflection is smaller than that for a small initial deflection. When the stress ratio is large, however, this is reversed in the region where the web slenderness ratio is large.

(3) When the web panel of aspect ratio 1 has the initial deflection of mode 2 or 3 in the $x$-direction, there exists the region where the fatigue strength becomes considerably smaller than the buckling strength depending on the magnitudes of the initial deflection and stress ratio.

(4) The residual stress reduces the fatigue strength. The degree of the reduction becomes smaller with the increases of the stress ratio and web slenderness ratio.

(5) Using the web slenderness ratio multiplied by the square root of the yielding stress, the buckling strength can be expressed independently of the yielding stress. But, even if such a parameter is used, the relation between fatigue strength and web slenderness ratio cannot be expressed independently of the yielding stress, because the fatigue strength of fillet welds subjected to secondary bending stress is almost constant regardless of an increase of the yielding stress.

\section{REFERENCES}

1) Yen, B.T. and Mueller, J.A. : Fatigue tests of large-sized welded plate girders, WRC, Bull. No.118, 1966.

2) Mueller, J.A. and Yen, B.T. : Girder web boundary stresses and fatigue, WRC, Bull. No.127, 1968.

3) Patterson, P.J., Corrade, J.A., Huang, J.S. and Yen, B.T. : Fatigue and static tests of two welded plate girders, WRC, Bull. No.155, 1970.

4) Toprac, A.A. and Natarajan, M. : Fatigue strength of hybrid plate girders, Proc. of ASCE, Vol.97, No.ST 4, pp.1203 1225, 1971.

5) Maeda, Y. : Ultimate static strength and fatigue behavior of longitudinally stiffened plate girders in bending, Proc. IABSE London Colloquium, pp.269 282, 1971.

6) AASHTO : Standard Specification for Highway Bridges, The American Association of State Highway and Transportation Officials, 12th edition, 1977.

7) Vincent, G.S. : Tentative criteria for load factor design of steel highway bridges, AISI, Bull. No.15, March, 1969.

8) BS5400 Part 10 : Code of Practice for Fatigue, British Standards Institution, 1980. 
9) Maeda, Y. and Okura, I. : Interaction between initial web deflection and fatigue crack initiation in thin-walled plate girders, The Design of Steel Bridges - Conference Discussion, edited by H.R. Evans, University College, Cardiff, pp.12.9 12.16, 1981.

10) Maeda, Y. and Okura, I. : Influence of initial deflection of plate girder web on fatigue crack initiation, Proc. of JSCE, No.319, pp.1 -11, 1982.

11) Gurney, T.R. : Fatigue of Welded Structures, Cambridge University Press, pp.96 116, 1968.

12) Maeda, Y. and Kawai, Y. : Fatigue strength of thin-walled welded hybrid girders in bending, Jour. of JWS, Vol.43, No.7, pp.736 742, 1974.

13) Maeda, Y. : Fatigue cracks of deep thin-walled plate girders, Proc. of Bridge Engineering Conference of TRB, Vol.1, pp.120 128, 1978.

14) Maeda, Y. and Okura, I. : Influence of initial deflection of plate girder webs on fatigue crack initiation, Eng. Struct., Vol.5, pp.58 66, Jan., 1983.

15) Marguerre, K. : Zur Theorie der gekrümmter Platte grosser Formänderung, Proc. 5th Int. Cong. Appl. Mech., Cambridge, pp.93 $101,1983$.

16) Maeda, Y. and Okura, I. : Study on finite out-of-plane deformation of rectangular plates subjected to compression and in-plane bending, Proc. of JSCE, No.320, pp.15 23, 1982.

17) Maeda, Y. and Okura I. : Effects of initial deflection on fatigue cracks due to out-of-plane deformation of thin plate, Proc. of JSCE, No.329, pp.1 11, 1983.

18) Maeda, Y. and Okura, I. : Analysis of deformation-induced fatigue of stiffened plate girder in bending, Anniversary Volume to Celebrate the Retirement of Prof. Ch. Massonnet, pp.255 264, March, 1984.

19) Timoshenko, S.P. and Gere, J.M. : Theory of Elastic Stability, 2nd edition, McGraw-Hill, pp.344 346, 1961.

20) Klöppel, K., Schmied, R. and Schubert, J. : Die Traglast mittig und außermittig gedrükter dünnwandiger Kastenträger unter Verwendung der nichtlinearen Beultheorie, Der Stahlbau, Vol.35, H.11, SS.321 337, 1966.

(Recieved December 14 1983) 